\title{
Presumed Melanoma of Unknown
} Primary Origin Metastatic to the Choroid Mimics Primary Uveal Melanoma

\author{
John M. Rieth ${ }^{a} \quad$ Randy Chris Bowen $^{b} \quad$ Mohammed M. Milhem ${ }^{c}$ \\ H. Culver Boldt ${ }^{b}$ Elaine M. Binkley ${ }^{b}$ \\ aDepartment of Internal Medicine, University of lowa, lowa, IA, USA; ${ }^{\circ}$ Department of \\ Ophthalmology and Visual Sciences, University of lowa, lowa, IA, USA; 'Department of \\ Medical Oncology, University of lowa, lowa, IA, USA
}

\author{
Keywords \\ Uveal melanoma Choroidal melanoma $\cdot$ Cutaneous melanoma $\cdot$ Choroidal metastasis
}

\section{Abstract}

We describe the case of a 69-year-old woman who presented with a decline in vision in the left eye and was found to have a choroidal lesion with clinical and echographic features concerning for primary uveal melanoma. Systemic imaging identified numerous metastases to the liver, kidneys, paratracheal lymph nodes, lung, and brain. The hepatic lesion was biopsied, and genetic analysis identified a Val600Glu (c.1799T>A) BRAF mutation, consistent with a cutaneous primary malignancy, although no primary tumor was identified. This case highlights that metastasis to the choroid is a rare presentation of nonuveal melanoma that can mimic primary uveal melanoma. Genetic analysis of tumor tissue can identify the origin of the melanoma and guide treatment options. Systemic imaging should be performed prior to intervention for choroidal neoplasms. 


\section{Introduction}

Metastasis of cutaneous and other nonocular melanomas to the uveal tract is rare, representing only $2 \%$ of uveal metastases. Of patients with nonocular melanomas that metastasize to the uveal tract, $96-98 \%$ of patients have a primary tumor identified prior to discovery of uveal metastasis, and it is rare for uveal metastasis to be the presenting feature $[1,2]$.

The most common ocular site of metastasis from cutaneous melanoma is the uvea, but other intraocular structures including the retina and vitreous may also be involved [2]. Uveal metastases in these cases are typically multifocal and flat, with a mean thickness of $1 \mathrm{~mm}$ [3], and demonstrate a more rapid growth pattern than primary uveal melanomas [2]. These metastases rarely present as amelanotic dome-shaped uveal masses [4]. Cutaneous and primary uveal melanoma are most importantly differentiated by their mutational signatures, with cutaneous melanomas frequently harboring mutations in $B R A F$ or NRAS, while primary uveal melanomas commonly carry mutations in BAP1, SF3B1, EIF1AX, GNA11, and/or GNAQ $[5,6]$. In $1.2-8.1 \%$ of patients with melanoma, no primary site is identified, and this is referred to as melanoma of unknown primary origin. The molecular signature in most of these cases is similar to those seen in cases of cutaneous melanoma with mutations in BRAF being quite common [7].

Here, we present a rare case of melanoma of unknown primary origin metastatic to the choroid, highlighting an uncommon mimic of primary uveal melanoma. We show that genetic analysis of tumor tissue can assist with identification of the origin of the melanoma and guide treatment options. We highlight the role of systemic imaging in establishing the diagnosis in this rare scenario.

\section{Case Report}

A 69-year-old woman was referred for further evaluation of a partially pigmented choroidal mass in the left eye. She reported a 1-month decline in vision in the left eye accompanied by photopsias. She had received annual dilated funduscopic examinations and had no history of choroidal nevus. Her only ocular history was that of bilateral LASIK and cataract extraction. She had no personal history of skin or other kinds of cancer. Family history included adenocarcinoma of the lung in her father, but no other malignancies. She reported a 2-month history of persistent cough that had been treated empirically with antibiotics without chest X-ray.

On ophthalmic examination, visual acuity was 20/30 in the right eye and 20/40 in the left eye. Anterior segment exam was unremarkable in both eyes. Posterior segment exam in the right eye was normal without choroidal lesions. In the left eye, located $2 \mathrm{~mm}$ superior to the optic nerve, there was an $8.0 \times 7.0 \times 2.6 \mathrm{~mm}$ mostly amelanotic choroidal lesion with a few areas of darker intrinsic pigment, prominent, diffuse orange pigment, and overlying subretinal fluid with no overlying drusen. Standardized ultrasonography showed a 2.6-mm thick, dome-shaped choroidal lesion with low internal reflectivity and intrinsic vascularity. Optical coherence tomography showed a choroidal lesion with a lobulated surface and overlying subretinal fluid (Fig. 1). At this point, the lesion was felt to be most consistent with a primary uveal melanoma. A baseline metastatic screening exam was performed with hepatic ultrasound which showed several hypoechoic lesions. MRI of the brain showed multiple intracranial and scalp nodules as well as the previously identified choroidal mass. CT of the chest, abdomen, and pelvis with iodinated contrast identified nodules in the kidneys, paratracheal lymph nodes, and lung. MRI of the abdomen with gadolinium was

\section{Karger'}



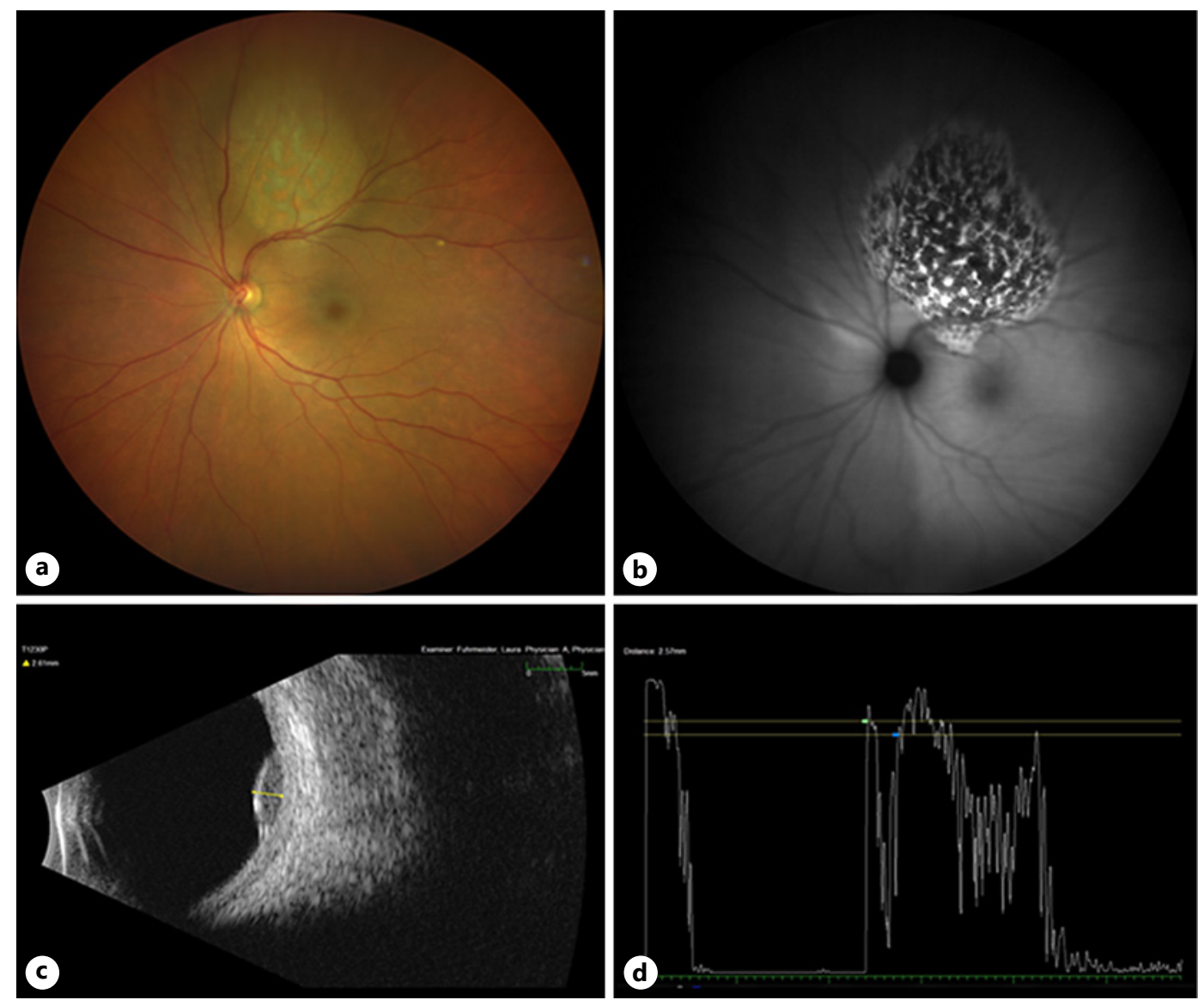

Fig. 1. Color fundus photograph of the left eye at presentation showed an $8.0 \times 7.0 \times 2.6 \mathrm{~mm}$ mostly amelanotic choroidal lesion with a few areas of darker intrinsic pigment, diffuse orange pigment, and surrounding subretinal fluid which tracked through the fovea (a). FAF at 6-week follow-up (note: there was no FAF taken at initial visit) showed intense hyperautofluorescence of lipofuscin overlying the tumor (b). B-scan ultrasound showed a dome-shaped choroidal mass with 2.6-mm thickness with corresponding A-scan ultrasound illustrating low internal reflectivity (c). Optical coherence tomography of the left eye demonstrated a lobulated choroidal mass extending into the superior macula with subretinal fluid extending beneath the fovea (d). FAF, fundus autofluorescence.

ordered to better characterize the hepatic lesions and demonstrated multiple hepatic nodules (Fig. 2). The patient underwent ultrasound-guided biopsy of one of the hepatic lesions which revealed tumor cells that were immunoreactive for SOX-10 and Melan-A. Melanoma mutation profiling revealed a Val600Glu (c.1799T $>A$ ) mutation in BRAF. There were no mutations in BAP1, SF3B1, EIF1AX, GNA11, or GNAQ. No cutaneous skin lesions were identified as the primary tumor.

Due to multiple brain metastases and the presumed nonuveal primary origin, the patient underwent palliative whole-brain and orbital radiation with a dose of 30 Gy over 10 fractions. Three weeks after completing whole-brain radiation, there was progression of the brain lesions, and the choroidal mass had grown to $12 \mathrm{~mm}$ in the largest basal diameter and 3.2-mm thickness (Fig. 3). The patient was started on pembrolizumab $200 \mathrm{mg}$ every 3 weeks, encorafenib $450 \mathrm{mg}$ daily, and binimetinib $45 \mathrm{mg}$ twice daily. Three weeks later, her visual acuity had improved from 20/40 to 20/30, and her tumor had decreased markedly to $12.0 \times 9.5 \times 1.2 \mathrm{~mm}$. Unfortunately, encorafenib and binimetinib were poorly 

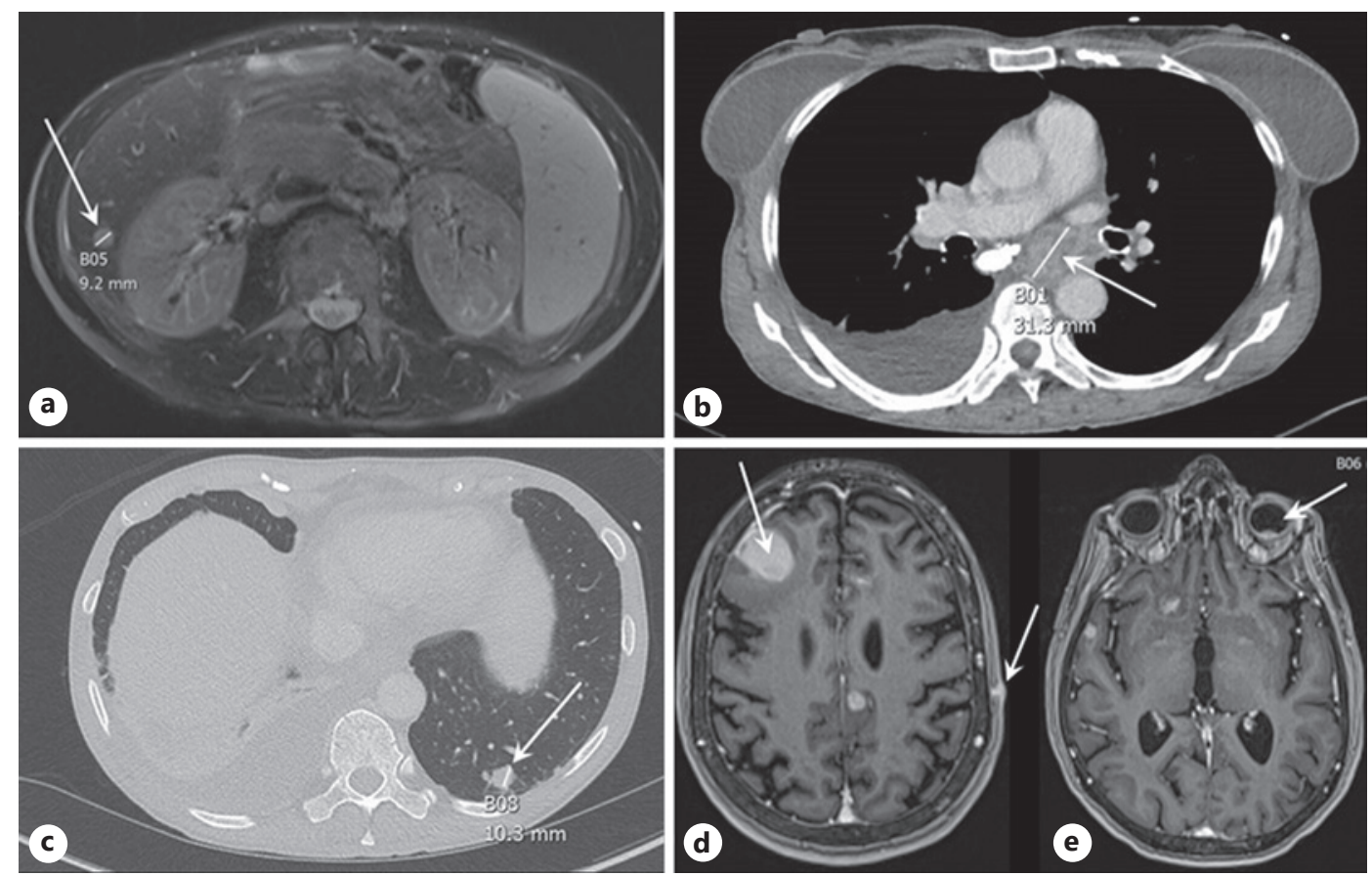

Fig. 2. MRI of metastases (white arrows) to the liver (a); CT imaging of enlarged paratracheal lymph nodes (b); lung metastasis (c); MRI showing metastasis of the intracranium and scalp (d) and choroid (e).
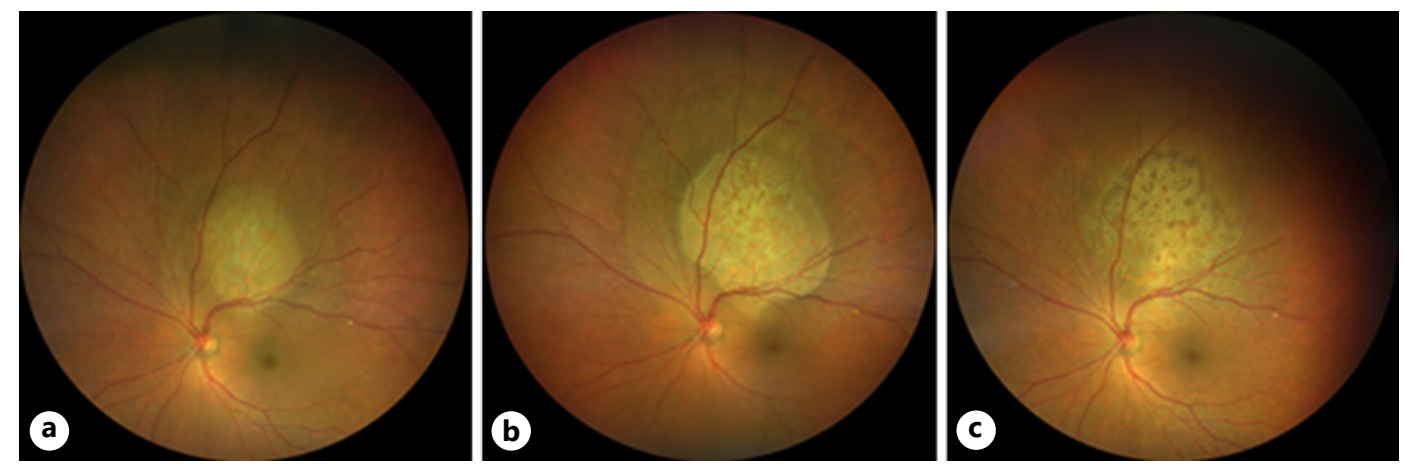

Fig. 3. a Color fundus photographs of the choroidal lesion at presentation, 6 weeks after initial presentation showing rapid growth (b), and 5 months after initial presentation with dramatic reduction in tumor size following treatment $(\mathbf{c})$.

tolerated and discontinued after 1 month. Due to the development of hydrocephalus, ventricular-peritoneal shunt was placed. Four months after presentation, the choroidal metastasis remained involuted, and her subretinal fluid had reabsorbed (Fig. 3, 4). Five months after presentation, a decreased dose of encorafenib was started (150 mg BID). Seven months after initial presentation, MRI brain and CT chest, abdomen, and pelvis showed stable or reduced lesions with no new masses. Unfortunately, during this time, she suffered from progressive cognitive decline. Despite initial systemic tumor reduction, 8 months after presentation, new brain lesions were detected, and she was provided with hospice care and passed away shortly thereafter (Fig. 4). 


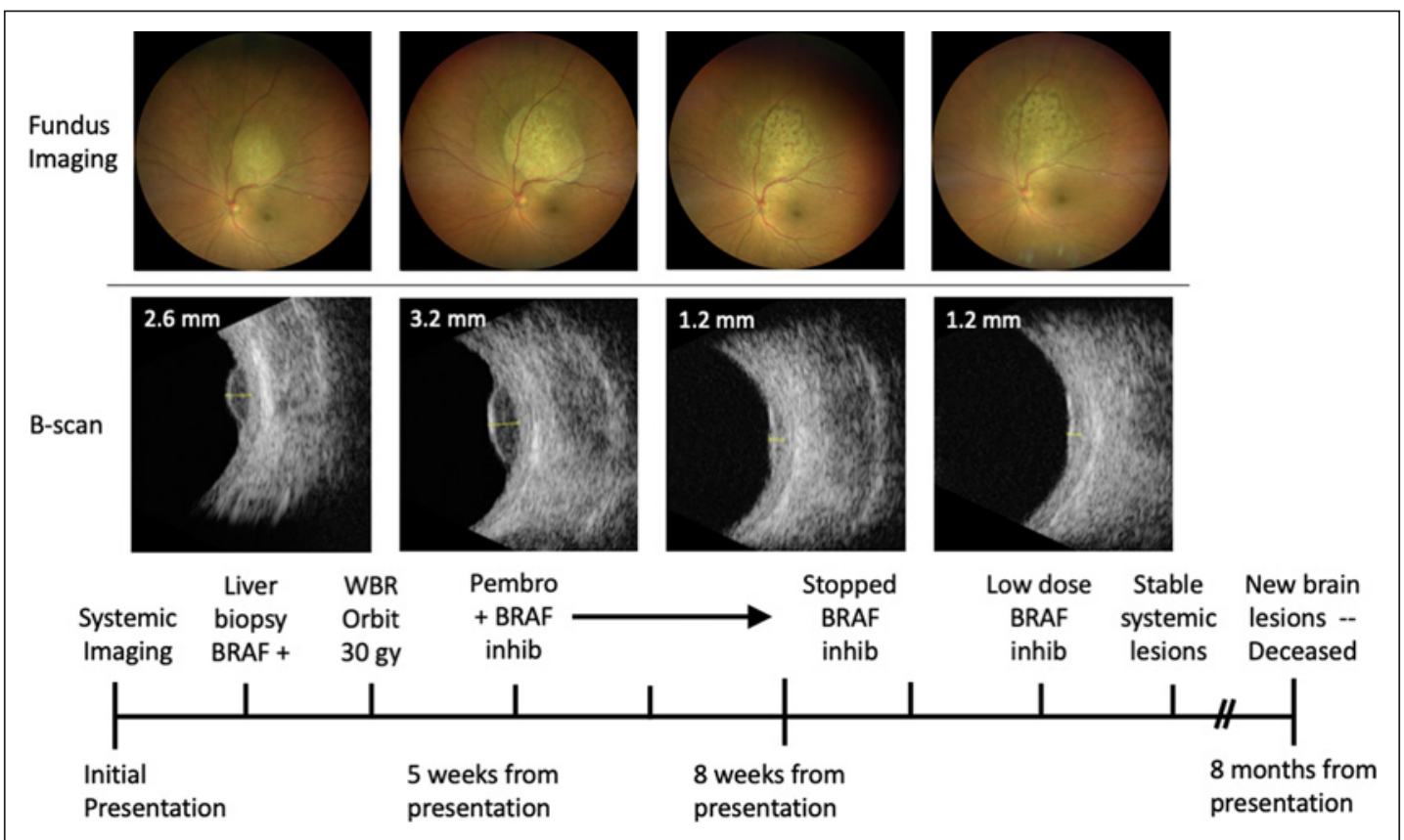

Fig. 4. Clinical timeline demonstrating rapid growth of choroidal tumor followed by rapid decline in tumor thickness with treatment. Pembro, pembrolizumab; Inhib, inhibitor; Gy, gray.

\section{Discussion}

Melanoma of unknown primary origin such as in this case represents only $1.2-8.1 \%$ of melanomas, and it is rare to present with uveal metastasis [7]. The dome shape, 2.6- $\mathrm{mm}$ thickness, low internal reflectivity, vascularity, and orange pigment of this lesion were all features more commonly seen in primary uveal melanoma. However, the wide metastatic distribution of the tumor identified on systemic imaging and the rapid growth of the lesion suggested an alternative diagnosis in this case.

Uveal melanoma classically metastasizes to the liver in about $90 \%$ of cases, and to the lungs in about $25 \%$. Metastasis to the brain from primary uveal melanoma is rare, occurring in approximately $5 \%$ of patients [8], and almost always in the setting of metastasis to the liver or lungs [9]. In contrast, metastasis to the brain is present in $30 \%$ of patients with cutaneous melanoma at diagnosis [10]. The presence of widespread brain metastasis in this case therefore suggested a higher probability of a nonuveal primary tumor.

Genetic analysis of the hepatic metastasis was key to the diagnosis in this case. Mutations in the BRAF gene are present in 40-60\% of melanomas of cutaneous origin, but are almost never present in primary uveal melanoma, making nonuveal melanoma much more likely in this case [11]. The possibility of simultaneous primary uveal and nonuveal melanoma was also considered in this patient, as this has been demonstrated in patients with the BAP1 tumor predisposition syndrome (BAP1-TPDS) [12] and familial atypical multiple mole melanoma [11,13]. Biopsy of the uveal lesion could have been performed to definitively rule this out; however, given the associated morbidity with biopsy in the setting of lower likelihood given the age of the patient and lack of personal or family history of BAP1-TPDS-associated tumors, the patient declined fineneedle aspiration biopsy of the uveal lesion. The clinical behavior of the lesion including the rapid growth and reduction in size after treatment with external beam radiation, PD-1 blockade, and BRAF/MEK inhibition was clinically consistent with melanoma of nonuveal origin. 
This lesion was initially presumed to be a primary uveal melanoma and illustrates that it is imperative to obtain systemic imaging prior to treating uveal neoplasms. Two percent of patients with primary uveal melanoma have metastatic disease at the time of diagnosis, and systemic imaging is indicated to rule out the presence of metastasis prior to treatment of the primary ocular lesion [14]. Furthermore, data from the Collaborative Ocular Melanoma Study (COMS) showed that ocular oncologists are over 99\% accurate in their diagnosis of primary uveal melanoma based upon clinical and echographic features [15]. However, this case demonstrates that there are instances where metastatic lesions can mimic primary uveal melanoma, and systemic imaging is key to identifying another primary malignancy. This case highlights importance of distinguishing between a primary uveal and metastatic cutaneous melanoma so that treatment can be appropriately tailored $[16,17]$.

In summary, metastasis to the uveal tract is an extremely rare presentation of melanoma of unknown primary origin. The clinical and systemic imaging features can help guide the management of patients with atypical presentations such as this case, and the ocular oncologist must always be aware of the possibility of this rare presentation. Genetic analysis of tumor tissue can assist with distinguishing between melanoma of primary cutaneous versus uveal origin.

\section{Acknowledgments}

This manuscript does not include any nonauthor contributors to acknowledge.

\section{Statement of Ethics}

The subject's daughter gave written informed consent for publication of this case as the patient had passed away. The IRB at the University of Iowa determined that this report does not meet the regulatory definition of human subject research and did not require formal IRB review since it is a single case report (see letter "nonhuman subjects research determination" in online suppl. material; for all online suppl. material, see www.karger.com/ doi/10.1159/000521199).

\section{Conflict of Interest Statement}

The authors have no conflicts of interest to declare.

\section{Funding Sources}

Randy Christopher Bowen is the recipient of a Heed ophthalmic fellowship.

\section{Author Contributions}

Each author fulfilled the ICGME criteria for authorship. J.R., R.B., M.M., C.B., and E.B. contributed to acquisition and analysis; J.R., R.B., M.M., C.B., and E.B. contributed to drafting and revising for intellectual content; J.R., R.B., M.M., C.B., and E.B. contributed to final approval of the version to be published.

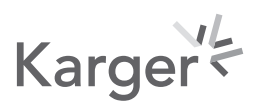


Rieth et al.: Nonuveal Melanoma Mimics Uveal Melanoma

\section{Data Availability Statement}

All data generated or analyzed during this study are included in this article. Further enquiries can be directed to the corresponding author.

\section{References}

1 Shields CL, Welch RJ, Malik K, Acaba-Berrocal LA, Selzer EB, Newman JH, et al. Uveal metastasis: clinical features and survival outcome of 2214 tumors in 1111 patients based on primary tumor origin. Middle East Afr J Ophthalmol. 2018;25(2):81.

2 Rosenberg C, Finger PT. Cutaneous malignant melanoma metastatic to the eye, lids, and orbit. Surv Ophthalmol. 2008;53(3):187-202.

3 Shields CL, Shields JA, Gross NE, Schwartz GP, Lally SE. Survey of 520 eyes with uveal metastases. Ophthalmology. 1997;104(8):1265-76.

4 Everett L, Damato BE, Bloomer MM, Palmer JD, Kao AA, Stewart JM, et al. Metastatic cutaneous melanoma presenting with choroidal metastasis simulating primary uveal melanoma. Ocul Oncol Pathol. 2019;5(2): 135-8.

5 Ticha I, Hojny J, Michalkova R, Kodet O, Krkavcova E, Hajkova N, et al. A comprehensive evaluation of pathogenic mutations in primary cutaneous melanomas, including the identification of novel loss-of-function variants. Sci Rep. 2019;9(1):17050-15.

6 Decatur CL, Ong E, Garg N, Anbunathan H, Bowcock AM, Field MG, et al. Driver mutations in uveal melanoma: associations with gene expression profile and patient outcomes. JAMA Ophthalmol. 2016;134(7):728-33.

7 Scott JF, Gerstenblith MR, Gerstenblith MR. Melanoma of unknown primary. Exon Publications; 2018. p. 99-116.

8 Willson JK, Albert DM, Diener-West M, McCaffrey L, Moy CS, Scully RE. Assessment of metastatic disease status at death in 435 patients with large choroidal melanoma in the collaborative ocular melanoma study (coms) coms report no. 15. Arch Ophthalmol. 2001;119(5):670-6.

9 Lorigan JG, Wallace S, Mavligit GM. The prevalence and location of metastases from ocular melanoma: imaging study in 110 patients. AJR Am J Roentgenol. 1991;157(6):1279-81.

10 Tawbi HA, Forsyth PA, Algazi A, Hamid O, Hodi FS, Moschos SJ, et al. Combined nivolumab and ipilimumab in melanoma metastatic to the brain. N Engl J Med. 2018;379(8):722-30.

11 Bellet RE, Shields JA, Soll DB, Bernardino EA. Primary choroidal and cutaneous melanomas occurring in a patient with the B-K mole syndrome phenotype. Am J Ophthalmol. 1980;89(4):567-70.

12 Chau C, van Doorn R, van Poppelen NM, van der Stoep N, Mensenkamp AR, Sijmons RH, et al. Families with BAP1-tumor predisposition syndrome in The Netherlands: path to identification and a proposal for genetic screening guidelines. Cancers. 2019;11(8):1114.

13 Abramson DH, Rodriguez-Sains RS, Rubman R. BK mole syndrome: cutaneous and ocular malignant melanoma. Arch Ophthalmol. 1980;98(8):1397-9.

14 Garg G, Finger PT, Kivelä TT, Simpson ER, Gallie BL, Saakyan S, et al. Patients presenting with metastases: stage IV uveal melanoma, an international study. Br J Ophthalmol. 2021.

15 Accuracy of diagnosis of choroidal melanomas in the Collaborative Ocular Melanoma Study. COMS report no. 1. Arch Ophthalmol. 1990;108(9):1268-73.

16 Rossi E, Pagliara MM, Orteschi D, Dosa T, Sammarco MG, Caputo CG, et al. Pembrolizumab as first-line treatment for metastatic uveal melanoma. Cancer Immunol ImmunotherImmunotherapy. 2019;68(7):1179-85.

17 Robert C, Schachter J, Long GV, Arance A, Grob JJ, Mortier L, et al. Pembrolizumab versus ipilimumab in advanced melanoma. N Engl J Med. 2015;372(26):2521-32.

\section{Karger'}

